\title{
ANÁLISE DA PRESENÇA DE OBESIDADE, COMPORTAMENTOS E FATORES DE RISCO CARDIOVASCULAR EM INDIVÍDUOS HIPERTENSOS
}

\author{
Beatriz Espanhol Garcia ${ }^{1}$, Carolina Takahashi ${ }^{1}$, Felipe Ribeiro ${ }^{1}$, Mariana Gutierrez ${ }^{1}$, Romy Buck Sonoda ${ }^{1}$, Laís Manata \\ Vanzella ${ }^{1}$, Gisele Carla Gonçalves ${ }^{1}$, Maria Isabela Haddad ${ }^{1}$, Luiz Carlos Marques Vanderlei ${ }^{1}$ \\ ${ }^{1}$ Universidade Estadual Paulista - UNESP, Departamento de Fisioterapia, Pro- Reitoria de Extensão Universitária - PROEX, \\ Presidente Prudente, SP. e-mail: Icmvanderlei@fct.unesp.br
}

\section{RESUMO}

A hipertensão arterial (HA) é o principal fator de risco (FR) para a morbidade e mortalidade precoces causadas por doenças cardiovasculares. O objetivo desse estudo é avaliar a presença de obesidade, comportamentos e FR cardiovascular em indivíduos hipertensos na FCT UNESP de Presidente Prudente. Foram avaliados três comportamentos de risco à saúde: tabagismo, etilismo e sedentarismo, e os fatores de risco avaliados foram obesidade, hipertensão arterial, dislipidemias e diabetes mellitus. O principal achado desse estudo é que há uma alta prevalência da obesidade, comportamentos e FR cardiovascular nesses indivíduos, aumentando a chance de desenvolver doenças cardiovasculares (DCV). Conclui-se que a presença de obesidade, comportamentos e FR cardiovascular em indivíduos hipertensos é elevada, sendo o histórico familiar de DCV e o sedentarismo, o comportamento e o fator de risco mais encontrados respectivamente.

Palavras-chave: hipertensão, fatores de risco, obesidade, doenças cardiovasculares, estilo de vida sedentário.

\section{ANALYSIS OF THE PRESENCE OF OBESITY, BEHAVIOUR AND CARDIOVASCULAR RISK FACTORS IN HYPERTENSIVE INDIVIDUALS}

\begin{abstract}
High blood pressure (hypertension) is a major risk factor (RF) for early morbidity and mortality caused by cardiovascular diseases. The aim of this study is to assess the presence of obesity, behavior and cardiovascular RF in hypertensive at FCT UNESP Presidente Prudente. We evaluated three health risk behaviors: smoking, drinking and physical inactivity, and the assessed risk factors were obesity, hypertension, dyslipidemia and diabetes mellitus. The main finding of this study is that there is a high prevalence of obesity, behavior and cardiovascular RF in these individuals, increasing the chance of developing cardiovascular disease (CVD). We conclude that the presence of obesity, behavior and cardiovascular RF in hypertensive individuals is high, being the family history of CVD and a sedentary lifestyle, the behavior and the risk factor most commonly found respectively
\end{abstract}

Keywords: hypertension, risk factors, obesity, cardiovascular diseases, sedentary lifestyle.

\section{INTRODUÇÃO}

A hipertensão arterial (HA) tem sido reconhecida como o principal fator de risco para a morbidade e mortalidade precoces causadas por doenças cardiovasculares sendo considerado um importante problema de saúde. É caracterizada por elevados e sustentados níveis de pressão arterial, e frequentemente está associada a alterações funcionais e ou estruturais dos órgãos alvo como coração, encéfalo, rins e vasos sanguíneos e também as alterações metabólicas ${ }^{1}$.

Estima-se que a cada ano morrem 7,6 milhões de pessoas em todo o mundo devido à hipertensão, sendo que mais da metade das vítimas encontram-se na faixa etária entre 45 e
69 anos e $80 \%$ dessas mortes ocorrem em países em desenvolvimento como o Brasil ${ }^{2}$.

A hipertensão arterial não ocorre de forma isolada, sendo que a maioria dos hipertensos apresenta outros fatores de risco (FR) cardiovasculares ${ }^{3}$. Os FR predispõem o surgimento das DCV, e vários estudos mostram que quando associados entre si e a outras condições, favorecem o aparecimento da hipertensão arterial $^{4}$. A obesidade é um importante fator de risco para o desenvolvimento da HA, estudos mostram redução dos níveis pressóricos quando há perda de peso ${ }^{5}$, e outros indicam que pelo menos dois terços da prevalência de HA podem ser diretamente atribuídos ao excesso de peso. Ainda, o risco de hipertensão arterial é cinco vezes maior entre 
pessoas obesas do que naquelas com peso normal ${ }^{6}$.

Além disso, comportamentos como sedentarismo, consumo de álcool e tabaco são considerados estilos de vida não saudáveis e podem potencializar o desenvolvimento de FR e contribuir para a elevação da pressão arterial ${ }^{7,8}$, no entanto esse tipo de comportamento pode ser alterado com mudanças nos hábitos de vida ${ }^{7}$.

Diante do exposto, o objetivo desse estudo foi avaliar a presença de obesidade e fatores de risco cardiovasculares em indivíduos hipertensos na Faculdade de Ciências e Tecnologia (FCT) UNESP de Presidente Pudente.

\section{MATERIAIS E MÉTODOS}

População

O estudo tem caráter epidemiológico de corte transversal e foram avaliados 15 sujeitos na Faculdade de Ciências e Tecnologia (FCT) UNESP de Presidente Pudente. Não foram incluídos no estudo pacientes que não apresentavam hipertensão arterial, e os que se recusaram a colaborar com a coleta de dados e aqueles que não compreenderam os questionários. $O$ estudo foi aprovado pelo Comitê de Ética em Pesquisa da FCT/UNESP (CAAE: 17442413.0.0000.5402).

\section{Coleta de Dados}

Para realizar a coleta de dados, uma equipe treinada visitou o local de trabalho de cada voluntário, sendo que inicialmente, foram explicados aos pacientes os objetivos e procedimentos do estudo. Após essa etapa, cada voluntário teve suas medidas antropométricas mensuradas e responderam algumas questões na forma de entrevista, sendo que os voluntários estavam livres para questionar acerca das dúvidas que surgiram durante todo o processo.

Foram avaliados três comportamentos de risco à saúde: tabagismo, etilismo e sedentarismo. $O$ tabagismo foi considerado presente quando o avaliado indicou ser fumante ou consumir ao menos um cigarro por dia ${ }^{9}$. O etilismo foi considerado para os entrevistados que reportaram o consumo diário de mais de 70 $\mathrm{ml}$ de qualquer tipo de bebida alcoólica ${ }^{10}$. E o sedentarismo foi considerado quando o indivíduo não relatava realizar nenhuma atividade física rotineira.

Os fatores de risco avaliados foram obesidade, hipertensão arterial, hipercolesterolemia, hipertrigliceridemia e diabetes mellitus. A obesidade foi considerada presente para os indivíduos que tiveram índice de massa corporal (IMC) acima de $30 \mathrm{~kg} / \mathrm{m}^{2}{ }^{11}$. Para obtenção dos valores de IMC, mensurou-se a massa corporal (balança Welmy, Brasil), estatura (estadiômetro Sanny, Brasil) e foi calculado o índice de massa corporal (IMC) (peso/altura ${ }^{2}$ ) de cada indivíduo em posição ortostática e vestindo roupas leves.

Posteriormente, foi realizada a mensuração da pressão arterial sendo esta aferida indiretamente por meio de esfigmomanômetro aneróide (WelchAlyn - Tycos, New York, USA), devidamente calibrado, e estetoscópio Littmann (Saint Paul, USA) com os voluntários sentados. Estes permaneceram em repouso por 5 minutos previamente à medida $\mathrm{e}$ duas aferições foram realizadas com intervalo de cinco minutos entre elas, sendo considerado para análise o menor valor. Ainda, dislipidemias e diabetes mellitus foram considerados presentes quando o indivíduo relatou ter alterações nos exames do último ano ou utilizou medicação para tal condição. Por fim, os indivíduos também foram questionados quanto ao histórico familiar de doenças cardiovasculares.

\section{Análise Estatística}

Para análise dos dados foi realizada a estatística descritiva e os resultados foram apresentados em valores de média, desviopadrões, números absolutos e percentuais.

\section{RESULTADOS}

Conforme está descrito na Tabela 1, a média do IMC dos indivíduos avaliados foi de $33,99 \pm 5,58 \mathrm{~kg} / \mathrm{m}^{2}$, indicando obesidade da maioria dos indivíduos.

O comportamento de risco com maior prevalência nos indivíduos hipertensos foi o sedentarismo, sendo este presente em 73,33\% dos indivíduos. Já o fator de risco mais prevalente foi à história familiar de doenças cardiovasculares prevalente em $80 \%$ da amostra. $O$ resultado das variáveis analisadas segue na Tabela 2. 
Tabela 1. Variáveis antropométricas da população apresentadas em média e desviopadrão.

\begin{tabular}{lc}
\hline \hline Variável & Valores \\
\hline Idade (anos) & $51,13 \pm 8,76$ \\
Peso $(\mathrm{Kg})$ & $101,09 \pm 24,15$ \\
Altura $(\mathrm{m})$ & $1,71 \pm 0,1$ \\
$\mathrm{IMC}\left(\mathrm{Kg} / \mathrm{m}^{2}\right)$ & $33,99 \pm 5,58$ \\
\hline \hline Legenda: $\mathrm{Kg}=$ & quilogramas; $\mathrm{m}=$ metros; IMC= \\
índice de massa corpórea; $\mathrm{m}^{2}=$ metros quadrados.
\end{tabular}

Tabela 2. Números absolutos e percentuais da distribuição dos comportamentos e fatores de risco da população estudada.

\begin{tabular}{lc}
\hline \hline Variáveis & $\begin{array}{c}\text { Prevalência ( } \mathbf{n = 1 5 )} \\
\mathrm{n}(\%)\end{array}$ \\
\hline Comportamentos & \\
Sedentarismo & $11(73,33)$ \\
Etilismo & $8(53,33)$ \\
Tabagismo & $4(26,66)$ \\
\hline Fator de Risco & \\
Dislipidemias & $3(20)$ \\
Diabetes mellitus & $2(13,33)$ \\
Obesidade & $15(25,0)$ \\
História familiar de DCV & $12(80)$ \\
\hline \hline
\end{tabular}

Legenda: $\mathrm{DCV}=$ doenças cardiovasculares

\section{DISCUSSÃO}

O principal achado desse estudo é que há uma alta presença da obesidade nesses indivíduos. Além desse importante fator de risco, a maior parte deles apresenta outros fatores de risco e comportamentos de risco, aumentando a chance de desenvolver DCV. Em um estudo semelhante $^{12}$ também observou elevada agregação de fatores de risco cardiovascular em indivíduos hipertensos.

Estudos relatam existência de relação direta da Pressão Arterial com a idade ${ }^{11}$. Em metalúrgicos do RJ e de SP a prevalência de HAS foi de $24,7 \%$ e a idade acima de 40 anos foi a variável que determinou maior risco para esta condição $^{11}$, corroborando com a média de idade dos hipertensos do presente estudo que foi de $51,13 \pm 8,76$ anos.

Todos os indivíduos apresentaram excesso de peso, sendo que $66,66 \%$ deles já apresentam obesidade e o restante encontra-se na faixa do sobrepeso. A literatura mostra que o excesso de peso se associa com maior prevalência de hipertensão arterial desde idades jovens, e que na vida adulta um incremento de $2,4 \mathrm{~kg} / \mathrm{m}^{2}$ no índice de massa corporal acarreta em maior risco de desenvolver a hipertensão ${ }^{11}$. Dessa forma, a forte associação existente entre a obesidade e a hipertensão arterial exige urgência de medidas educativas que sejam capazes de atuar sobre os fatores de risco que podem determinar a prevalência da hipertensão arterial, orientando os indivíduos a aderirem a um estilo de vida mais saudável ${ }^{13}$, interferindo também na redução dos comportamentos de risco desses indivíduos.

No nosso estudo o comportamento de risco mais prevalente foi o sedentarismo $(73,33 \%)$, que contribui de forma positiva para o aumento da pressão arterial. Estudos referem que a atividade física reduz a incidência de hipertensão arterial além de reduzir a mortalidade e os riscos de desenvolver doenças cardiovasculares e favorecer um envelhecimento saudáve ${ }^{3,11}$. Conceição et al. também observou elevada prevalência de altos níveis pressóricos em um grupo de servidores públicos, além de outros fatores de risco cardiovasculares, o que sugere a necessidade da adoção de medidas educativas preventivas e terapêuticas em relação as doenças cardiovasculares, corroborando com nosso estudo ${ }^{14}$.

Apenas $4 \%$ da população referiu uso de tabaco, e porcentagem semelhante foi encontrada em um grupo de 88 mulheres hipertensas na cidade de São Luís-MA $(3,41 \%)^{15}$. Embora o tabagismo tenha sido o comportamento de risco menos encontrado nessas populações, ele é altamente prejudicial, visto que mediado pela nicotina age como um agonista adrenérgico, promovendo a liberação local e sistêmica de catecolaminas causando um aumento agudo da pressão arterial e da frequência cardíaca ${ }^{16}$.

Quanto aos fatores de rico cardiovascular, o mais encontrado foi história familiar de doenças cardiovasculares estando presente em $80 \%$ da população, da mesma forma Santos et al. encontrou elevada prevalência de história familiar de DCV em hipertensos no nordeste brasileiro ${ }^{17}$.

A diabetes mellitus foi encontrado em apenas $13,33 \%$, entretanto merece atenção, visto que a coexistência de hipertensão e diabetes aumenta o risco para complicações micro e macrovasculares, predispondo os indivíduos a insuficiência cardíaca congestiva, doença 
coronariana e cerebrovascular, insuficiência arterial periférica e outros ${ }^{18}$.

Em relação às limitações do estudo, a amostra foi composta por um número relativamente reduzido de participantes, podendo fazer com que os nossos resultados possam apenas ser aplicados a grupos semelhantes aos usados neste estudo.

Este estudo possui implicações clínicas positivas para os profissionais da área da saúde, visto que identificar a prevalência de obesidade e fatores de risco cardiovasculares em indivíduos hipertensos é importante, pois torna-se possível a adoção de medidas preventivas para prevenir o risco que a associação destes podem causar na saúde dos indivíduos. Modificações no estilo de vida não são inatingíveis, têm o potencial de prevenir a hipertensão, são efetivas em diminuir a pressão arterial e podem reduzir outros fatores de risco cardiovascular a um custo pequeno e com risco mínimo ${ }^{12}$.

\section{CONCLUSÕES}

A partir dos achados, conclui-se que a presença de obesidade, comportamentos e fatores de risco cardiovascular em indivíduos hipertensos são elevados, sendo o histórico familiar de DCV e o sedentarismo, o fator de risco e o comportamento mais encontrados respectivamente.

\section{CONFLITO DE INTERESSE}

Os autores declaram não haver qualquer potencial de conflito de interesse que possa interferir na imparcialidade deste trabalho científico.

\section{REFERÊNCIAS}

1. Ávila A, Tavares A, Machado CA, Campana EMG, Lessa I, Krieger JE et al. VI Diretrizes Brasileiras de Hipertensão, Conceituação, Epidemiologia e Prevenção Primária. Rev Bras Hipertens. 2010;17(1):710.

2. Malachias, MVB. VI Diretrizes Brasileiras de Hipertensão, Palavra do Presidente. Rev Bras Hipertens. 2010;17(1):2-3.

3. Costa MFFL, Peixoto SV, César CC, Malta DC, Moura EC. Comportamentos em saúde entre idosos hipertensos, Brasil, 2006. Rev Saúde Pública. 2009;43(2):18-26.

DOI: http://dx.doi.org/10.1590/\$0034-9102009000900004
4. Pansani AP, Anequini IP, Vanderlei LCM, Tarumoto $\mathrm{MH}$. Prevalência de fatores de risco para doenças coronarianas em idosas frequentadoras de um programa "Universidade Aberta à Terceira Idade". Arq Ciênc Saúde. 2005;12(1):27-31.

5. Sacks FM, Bray GA et al. Comparison of weight-loss diets with different compositions of fat, protein, and carbohydrates. N Engl J Med 2009;360(9):859-73.

6. Buckland G, Bach A, Serra-Majem L. Obesity and the Mediterranean diet: a systematic review of observational and intervention studies. Obes Ver. 2008;9:582-93.

7. Bernardo AFB, Fernandes RA, Silva AKF, Valenti VE, Pastre CM, Vanderlei LCM. Influence of risk behavior aggregation in different categories of physical activity on the occurrence of cardiovascular risk factors. Int Arch Med. 2013;6:26.

8. Braunwald E, Fauci AS, Hauser SL, Longo DL, Kasper DL, Jameson JL. Harrison Medicina Interna 17.ed. Rio de Janeiro: McGraw-Hill; 2008:1549-62.

9. A clinical practice guideline for treating tobacco use and dependence: A US Public Health Service report. The Tobacco Use and Dependence Clinical Practice Guideline Panel, Staff, and Consortium Representatives. JAMA 2000:283(24):3244-54.

10. Jozala E, Infante S, Marchini JS, Okano N. Alcoolismo, tabagismo e carcinoma epidermóide de terço médio do esôfago: estudo tipo caso-controle. Rev Saúde Pública 1983;17(3):221-5.

11. Diretrizes brasileiras de obesidade 2009/2010 / ABESO - Associação Brasileira para o Estudo da Obesidade e da Síndrome Metabólica. Itapevi: AC Farmacêutica; 2009;3.

12. Renner SBA, Franco RR, Berlezi EM, Bertholo SC. Associação da hipertensão arterial com fatores de riscos cardiovasculares em hipertensos de ljuí, RS. RBAC. 2008;40(4):261-66.

13. Jardim PCBV, Gondim MRP, Monego ET, Moreira HG, Vitorino PVO, Souza WKSB et al. Hipertensão arterial e alguns fatores de risco em uma capital brasileira. Arq Bras Cardiol. 2007;88(4):452-7. DOI: $\quad$ http://dx.doi.org/10.1590/S0066-

\section{$\underline{782 \times 2007000400015}$}

14. Conceição TV, Gomes FA, Tauil PL, Rosa TT. Valores de pressão arterial e suas associações com fatores de risco cardiovasculares em servidores da Universidade de Brasília. Arq Bras Cardiol. 2006;86(1):26-3.

15. Nascimento JS, Gomes B, Sardinha AHL. Fatores de risco modificáveis para doenças cardiovasculares em mulheres com hipertensão arterial. Rev Rene. 2011;12(4):709-15.

16. Benowitz NL. Cigarette smoking and nicotine addiction. Med Clin North Am. 1992;76:415-38.

17. Santos JC, Moreira TMM. Fatores de risco e complicações em hipertensos/diabéticos de uma regional sanitária do nordeste brasileiro. Rev Esc Enferm USP. 2012;46(5):1125-32. DOI: http://dx.doi.org/10.1590/S0080-2342012000500013 
18. Faria AN, Zanella MT, Kohlman O, Ribeiro AB. Tratamento de diabetes e hipertensão no paciente obeso. Arq Bras Endocrinol Metab. 2002;46(2):137-42.

DOI: http://dx.doi.org/10.1590/S0004-
Recebido para publicação em 28/08/2015 Revisado em 02/09/2015

Aceito em 05/09/2015

\section{$\underline{27302002000200004}$}

